\title{
ON SOME SUMMABILITY FACTORS OF INFINITE SERIES
}

\author{
W. T. SULAIMAN
}

(Communicated by Andrew M. Bruckner)

\begin{abstract}
A new theorem concerning some summability factors of infinite series is proved. Other results, some of them known, are also deduced.
\end{abstract}

\section{INTRODUCTION}

Let $\sum a_{n}$ be an infinite series of partial sums $s_{n}$. Let $\sigma_{n}^{\delta}$ and $\eta_{n}^{\delta}$ denote the $n$th Cesàro mean of order $\delta(\delta>-1)$ of the sequences $\left\{s_{n}\right\}$ and $\left\{n a_{n}\right\}$ respectively. The series $\sum a_{n}$ is said to be absolutely summable $(C, \delta)$ with index $k$, or simply summable $|C, \delta|_{k}, k \geq 1$, if

$$
\sum_{n=1}^{\infty} n^{k-1}\left|\sigma_{n}^{\delta}-\sigma_{n-1}^{\delta}\right|^{k}<\infty
$$

or equivalently

$$
\sum_{n=1}^{\infty} n^{-1}\left|\eta_{n}\right|^{k}<\infty
$$

Let $\left\{p_{n}\right\}$ be a sequence of positive real constants such that

$$
P_{n}=\sum_{v=0}^{n} p_{v} \rightarrow \infty \quad \text { as } n \rightarrow \infty \quad\left(P_{-1}=p_{-1}=0\right) \text {. }
$$

The series $\sum a_{n}$ is said to be summable $\left|\bar{N}, p_{n}\right|_{k}, k \geq 1$, if

$$
\sum_{n=1}^{\infty}\left(P_{n} / p_{n}\right)^{k-1}\left|T_{n}-T_{n-1}\right|^{k}<\infty \quad \text { (Bor [1]), }
$$

where

$$
T_{n}=P_{n}^{-1} \sum_{v=0}^{n} p_{v} s_{v}
$$

If we take $p_{n}=1$, then $\left|\bar{N}, p_{n}\right|_{k}$ summability is equivalent to $|C, 1|_{k}$ summability. $\left|\bar{N}, p_{n}\right|_{1}$ is the same as $\left|\bar{N}, p_{n}\right|$. In general, the two summability methods $|C, \delta|_{k}$ and $\left|\bar{N}, p_{n}\right|_{k}$ are not comparable.

Received by the editors February 7, 1990.

1991 Mathematics Subject Classification. Primary 40G99. 
Here we give the following new definition: Let $\left\{\varphi_{n}\right\}$ be any sequence of positive real constants. The series $\sum a_{n}$ is said to be summable $\left|\bar{N}, p_{n}, \varphi_{n}\right|_{k}$, $k \geq 1$, if

$$
\sum_{n=1}^{\infty} \varphi_{n}^{k-1}\left|T_{n}-T_{n-1}\right|^{k}<\infty
$$

Clearly $\left|\bar{N}, p_{n}, P_{n} / p_{n}\right|_{k}=\left|\bar{N}, p_{n}\right|_{k},\left|\bar{N}, p_{n}, 1\right|_{1}=\left|\bar{N}, p_{n}\right|$, and $|\bar{N}, 1, n|_{k}=$ $|C, 1|_{k}$. The following two results are due to Bor:

Theorem 1. Let $\left\{p_{n}\right\}$ be a sequence of positive real constants such that as $n \rightarrow \infty$

(i) $n p_{n}=0\left(P_{n}\right)$,

(ii) $P_{n}=0\left(n p_{n}\right)$.

If $\sum a_{n}$ is summable, $|C, 1|_{k}$, then it is also summable $\left|\bar{N}, p_{n}\right|_{k}, k \geq 1$.

Theorem 2. Let $\left\{p_{n}\right\}$ be a sequence of positive real constants such that it satisfies (1.1). If $\sum a_{n}$ is summable $\left|\bar{N}, p_{n}\right|_{k}$, then it is also summable $|C, 1|_{k}$.

We prove the following:

Theorem 3. Let $\left\{p_{n}\right\},\left\{q_{n}\right\}$, and $\left\{\varphi_{n}\right\}$ be sequences of positive real constants such that $\left\{\varphi_{n} q_{n} / Q_{n}\right\}$ is nonincreasing. Let $t_{n}$ denote the $\left(\bar{N}, p_{n}\right)$-mean of the series $\sum a_{n}$. If

$$
\begin{gathered}
\sum_{n=1}^{\infty}\left(\frac{P_{n}}{p_{n}}\right)^{k}\left(\frac{q_{n}}{Q_{n}}\right)^{k} \varphi_{n}^{k-1}\left|\varepsilon_{n}\right|^{k}\left|\Delta t_{n-1}\right|^{k}<\infty, \\
\sum_{n=1}^{\infty} \varphi_{n}^{k-1}\left|\varepsilon_{n}\right|^{k}\left|\Delta t_{n-1}\right|^{k}<\infty,
\end{gathered}
$$

and

$$
\sum_{n=1}^{\infty}\left(\frac{P_{n}}{p_{n}}\right)^{k} \varphi_{n}^{k-1}\left|\Delta \varepsilon_{n}\right|^{k}\left|\Delta t_{n-1}\right|^{k}<\infty
$$

then the series $\sum a_{n} \varepsilon_{n}$ is summable $\left|\bar{N}, q_{n}, \varphi_{n}\right|_{k}, k \geq 1$, where $\Delta f_{n}=f_{n}-f_{n+1}$ and $Q_{n}=\sum_{v=0}^{n} q_{v} \rightarrow \infty$ as $n \rightarrow \infty \quad\left(Q_{-1}=q_{-1}=0\right)$.

\section{Proof of TheOREM 3}

Let $T_{n}$ be the $\left(\bar{N}, q_{n}\right)$-mean of the series $\sum a_{n} \varepsilon_{n}$. Then we have

$$
T_{n}=\frac{1}{Q_{n}} \sum_{v=0}^{n} q_{v} \sum_{r=0}^{v} a_{r} \varepsilon_{r}=\frac{1}{Q_{n}} \sum_{v=0}^{n}\left(Q_{n}-Q_{v-1}\right) a_{v} \varepsilon_{v}
$$


Hence

$$
T_{n}-T_{n-1}=\frac{q_{n}}{Q_{n} Q_{n-1}} \sum_{v=1}^{n} Q_{v-1} a_{v} \varepsilon_{v}
$$

Abel's transformation gives

$$
\begin{aligned}
T_{n}-T_{n-1}= & \frac{q_{n}}{Q_{n} Q_{n-1}}\left\{\sum_{v=1}^{n-1}\left(\sum_{r=1}^{v} P_{r-1} a_{r}\right) \Delta\left(P_{v-1}^{-1} Q_{v-1} \varepsilon_{v}\right)\right. \\
& \left.\quad+\left(\sum_{r=1}^{n} P_{r-1} a_{r}\right) P_{n-1}^{-1} Q_{n-1} \varepsilon_{n}\right\} \\
= & \frac{q_{n}}{Q_{n} Q_{n-1}} \sum_{v=1}^{n-1}\left\{-Q_{v-1} \varepsilon_{v} \Delta t_{v-1}+\frac{P_{v-1}}{p_{v}} q_{v} \varepsilon_{v} \Delta t_{v-1}-\frac{P_{v-1}}{p_{v}} Q_{v} \Delta \varepsilon_{v} \Delta t_{v-1}\right\} \\
& -\left(\frac{P_{n}}{p_{n}}\right)\left(\frac{q_{n}}{Q_{n}}\right) \varepsilon_{n} \Delta t_{n-1} \\
= & T_{n, 1}+T_{n, 2}+T_{n, 3}+T_{n, 4}, \text { say. }
\end{aligned}
$$

To prove the theorem, it is sufficient, by Minkowski's inequality, to show that

$$
\sum_{n=1}^{\infty} \varphi_{n}^{k-1}\left|T_{n, r}\right|^{k}<\infty, \quad r=1,2,3,4
$$

Applying Hölder's inequality,

$$
\begin{aligned}
\sum_{n=2}^{m+1} & \varphi_{n}^{k-1}\left|T_{n, 1}\right|^{k}=\sum_{n=2}^{m+1} \varphi_{n}^{k-1}\left(\frac{q_{n}}{Q_{n} Q_{n-1}}\right)^{k}\left|\sum_{v=1}^{n-1}-\frac{Q_{v-1}}{q_{v}} q_{v} \varepsilon_{v} \Delta t_{v-1}\right|^{k} \\
\leq & \sum_{n=2}^{m+1} \varphi_{n}^{k-1}\left(\frac{q_{n}}{Q_{n}}\right)^{k} \frac{1}{Q_{n-1}} \\
& \times \sum_{v=1}^{n-1}\left(\frac{Q_{v}}{q_{v}}\right)^{k} q_{v}\left|\varepsilon_{v}\right|^{k}\left|\Delta t_{v-1}\right|^{k}\left\{\frac{1}{Q_{n-1}} \sum_{v=1}^{n-1} q_{v}\right\}^{k-1} \\
\leq & 0(1) \sum_{v=1}^{m}\left(\frac{Q_{v}}{q_{v}}\right)^{k} q_{v}\left|\varepsilon_{v}\right|^{k}\left|\Delta t_{v-1}\right|^{k} \sum_{n=v+1}^{m+1}\left(\frac{\varphi_{n} q_{n}}{Q_{n}}\right) \frac{q_{n}}{Q_{n} Q_{n-1}} \\
\leq & 0(1) \sum_{v=1}^{m} \varphi_{v}^{k-1} Q_{v}\left|\varepsilon_{v}\right|^{k}\left|\Delta t_{v-1}\right|^{k} \sum_{n=v+1}^{m+1} \frac{q_{n}}{Q_{n} Q_{n-1}} \\
\leq & 0(1) \sum_{v=1}^{m} \varphi_{v}^{k-1}\left|\varepsilon_{v}\right|^{k}\left|\Delta t_{v-1}\right|^{k},
\end{aligned}
$$




$$
\begin{aligned}
& \sum_{n=2}^{m+1} \varphi_{n}^{k-1}\left|T_{n, 2}\right|^{k}=\sum_{n=2}^{m+1} \varphi_{n}^{k-1}\left(\frac{q_{n}}{Q_{n} Q_{n-1}}\right)^{k}\left|\sum_{v=1}^{n-1}-\frac{P_{v-1}}{p_{v}} q_{v} \varepsilon_{v} \Delta t_{v-1}\right|^{k} \\
& \leq \sum_{n=2}^{m+1} \varphi_{n}^{k-1}\left(\frac{q_{n}}{Q_{n}}\right)^{k} \frac{1}{Q_{n-1}} \sum_{v=1}^{n-1}\left(\frac{P_{v}}{p_{v}}\right)^{k} q_{v}\left|\varepsilon_{v}\right|^{k}\left|\Delta t_{v-1}\right|^{k}\left\{\frac{1}{Q_{n-1}} \sum_{v=1}^{n-1} q_{v}\right\}^{k-1} \\
& \leq 0(1) \sum_{v=1}^{m}\left(\frac{P_{v}}{p_{v}}\right)^{k} q_{v}\left|\varepsilon_{v}\right|^{k}\left|\Delta t_{v-1}\right|^{k} \sum_{n=v+1}^{m+1}\left(\frac{\varphi_{n} q_{n}}{Q_{n}}\right)^{k-1} \frac{q_{n}}{Q_{n} Q_{n-1}} \\
& \leq 0(1) \sum_{v=1}^{m} \varphi_{v}^{k-1}\left(\frac{P_{v}}{p_{v}}\right)^{k}\left(\frac{q_{v}}{Q_{v}}\right)^{k-1} q_{v}\left|\varepsilon_{v}\right|^{k}\left|\Delta t_{v-1}\right|^{k} \sum_{n=v+1}^{m+1} \frac{q_{n}}{Q_{n} Q_{n-1}} \\
& \leq 0(1) \sum_{v=1}^{m} \varphi_{v}^{k-1}\left(\frac{P_{v}}{p_{v}}\right)^{k}\left(\frac{q_{v}}{Q_{v}}\right)^{k}\left|\varepsilon_{v}\right|^{k}\left|\Delta t_{v-1}\right|^{k}, \\
& \sum_{n=2}^{m+1} \varphi_{n}^{k-1}\left|T_{n, 3}\right|^{k}=\sum_{n=2}^{m+1} \varphi_{n}^{k-1}\left(\frac{q_{n}}{Q_{n} Q_{n-1}}\right)^{k}\left|\sum_{v=1}^{n-1}-\frac{P_{v-1}}{p_{v}} \frac{Q_{v}}{q_{v}} q_{v} \Delta \varepsilon_{v} \Delta t_{v-1}\right|^{k} \\
& \leq \sum_{n=2}^{m+1} \varphi_{n}^{k-1}\left(\frac{q_{n}}{Q_{n}}\right)^{k} \frac{1}{Q_{n-1}} \sum_{v=1}^{n-1}\left(\frac{P_{v}}{p_{v}}\right)^{k}\left(\frac{Q_{v}}{q_{v}}\right)^{k} q_{v}\left|\Delta \varepsilon_{v}\right|^{k}\left|\Delta t_{v-1}\right|^{k} \\
& \times\left\{\frac{1}{Q_{n-1}} \sum_{v=1}^{n-1} q_{v}\right\}^{k-1} \\
& \leq 0(1) \sum_{v=1}^{m}\left(\frac{P_{v}}{p_{v}}\right)^{k}\left(\frac{Q_{v}}{q_{v}}\right)^{k} q_{v}\left|\Delta \varepsilon_{v}\right|^{k}\left|\Delta t_{v-1}\right|^{k} \sum_{n=v+1}^{m+1}\left(\frac{\varphi_{n} q_{n}}{Q_{n}}\right)^{k-1} \\
& \times \frac{q_{n}}{Q_{n} Q_{n-1}} \\
& \leq 0(1) \sum_{v=1}^{m} \varphi_{v}^{k-1}\left(\frac{P_{v}}{p_{v}}\right)^{k} Q_{v}\left|\Delta \varepsilon_{v}\right|^{k}\left|\Delta t_{v-1}\right|^{k} \sum_{n=v+1}^{m+1} \frac{q_{n}}{Q_{n} Q_{n-1}} \\
& \leq 0(1) \sum_{v=1}^{m} \varphi_{v}^{k-1}\left(\frac{P_{v}}{p_{v}}\right)^{k}\left|\Delta \varepsilon_{v}\right|^{k}\left|\Delta t_{v-1}\right|^{k} \text {, and } \\
& \sum_{n=1}^{m} \varphi_{n}^{k-1}\left|T_{n, 4}\right|^{k}=\sum_{n=1}^{m} \varphi_{n}^{k-1}\left|-\left(\frac{P_{n}}{p_{n}}\right)\left(\frac{q_{n}}{Q_{n}}\right) \varepsilon_{n} \Delta t_{n-1}\right|^{k} \\
& \leq 0(1) \sum_{n=1}^{m} \varphi_{n}^{k-1}\left(\frac{P_{n}}{p_{n}}\right)^{k}\left(\frac{q_{n}}{Q_{n}}\right)^{k}\left|\varepsilon_{n}\right|^{k}\left|\Delta t_{n-1}\right|^{k}
\end{aligned}
$$

This completes the proof of the theorem.

\section{ApPlications}

Corollary 1. If

(i) $p_{n} Q_{n}=0\left(P_{n} q_{n}\right)$,

(ii) $P_{n} q_{n}=0\left(p_{n} Q_{n}\right)$, 
then the series $\sum a_{n}$ is summable $\left|\bar{N}, q_{n}, \varphi_{n}\right|_{k}$, whenever it is summable $\left|\bar{N}, p_{n}, \varphi_{n}\right|_{k}, k \geq 1$, and $\varphi_{n}=0\left(\phi_{n}\right)$.

The proof follows from Theorem 3 by putting $\varepsilon_{n}=1$.

Corollary 2. If (3.1) is satisfied, then the series $\sum a_{n}$ is summable $\left|\bar{N}, q_{n}\right|_{k}$ whenever it is summable $\left|\bar{N}, p_{n}\right|_{k}, k \geq 1$.

The proof follows from Corollary 1 by putting $\varphi_{n}=Q_{n} / q_{n}=0\left(P_{n} / p_{n}\right)$.

Corollary 3 (Theorems 1 and 2). If (1.1) is satisfied, then the series $\sum a_{n}$ is summable $|C, 1|_{k}$ if and only if it is summable $\left|\bar{N}, p_{n}\right|_{k}, k \geq 1$.

Proof. $(\Rightarrow)$ follows from Corollary 2 by putting $p_{n}=1$.

$(\Leftarrow)$ follows from Corollary 2 by putting $q_{n}=1$.

Corollary 4. If
(i) $\frac{P_{n} q_{n}}{p_{n} Q_{n}} \varepsilon_{n}=0(1)$,
(ii) $\varepsilon_{n}=0(1)$,
(iii) $\frac{P_{n}}{p_{n}} \Delta \varepsilon_{n}=0(1)$,

then the series $\sum a_{n} \varepsilon_{n}$ is summable $\left|\bar{N}, q_{n}, \varphi_{n}\right|_{k}$, whenever $\sum a_{n}$ is summable $\left|\bar{N}, p_{n}, \varphi_{n}\right|_{k}, k \geq 1$.

The proof follows from Theorem 3 .

Corollary 5. If
(i) $\varepsilon_{n}=0(1)$,
(ii) $\Delta \varepsilon_{n}=0(1 / n)$,

then the series $\sum a_{n} \varepsilon_{n}$ is summable $|C, 1|_{k}$, whenever $\sum a_{n}$ is summable $|C, 1|_{k}, k \geq 1$.

The proof follows from Corollary 4 by putting $p_{n}=q_{n}=1, \varphi_{n}=n$.

\section{ACKNOWLEDGMENTS}

The author is very grateful to the referee for his interesting comments.

\section{REFERENCES}

1. H. Bor, On two summability methods, Math. Proc. Cambridge Philos. Soc. 97 (1985), 147149.

2. __ A note on two summability methods, Proc. Amer. Math. Soc. 98 (1986), 81-84.

Department of Applied Sciences, College of Technological Studies, P. O. Box 42325, 70654 KUWAIT 